\title{
The Real World of Gout
}

Gout is a common cause of acute painful arthritis with a prevalence of $3.9 \%$ of adults in the United States, affecting an estimated 8.3 million people ${ }^{1}$. Gout prevalence increases with age, affecting $12.6 \%$ of those aged 80 and over ${ }^{1}$. Untreated and undertreated gout progresses to a chronic disabling arthropathy in a significant number of patients ${ }^{2}$. An increasing proportion of patients with gout have complex profiles of comorbidities and long prescription medication lists further complicating their management ${ }^{3}$.

In this setting the recent publication of the American College of Rheumatology (ACR) guidelines for the management of gout are a welcome addition to the resources at the disposal of practicing clinicians to assist in the management of this group of potentially complex patients ${ }^{4,5}$. The ACR guidelines recommend probenecid as an alternative first-line pharmacological urate-lowering therapy for those with a history of contraindication or intolerance to a xanthine oxidase inhibitor and with a creatinine clearance of $50 \mathrm{ml} / \mathrm{min}$ or more ${ }^{4}$.

Probenecid is a uricosuric agent that lowers serum urate by inhibiting renal tubular reabsorption of uric acid. Probenecid is infrequently prescribed as a urate-lowering therapy in many parts of the world including the United States and Europe ${ }^{6,7}$. The study reported by Pui, et al in this issue of The Journal is of considerable interest ${ }^{8}$.

Pui, et al report their real world experience of probenecid usage in 57 patients attending a rheumatology clinic in New Zealand. The proportion of patients receiving probenecid was relatively high, at $10.9 \%$, perhaps influenced by the present lack of availability of febuxostat in this market. The population described appear to have difficult-to-treat gout, with a high proportion (60\%) with tophaceous gout and a mean baseline serum urate of $600 \mu \mathrm{mol} / 1$. Half the patients started probenecid because of inadequate serum urate control while taking allopurinol (albeit at a mean dose of $362 \mathrm{mg}$ ); in these patients mean serum urate on allopurinol treatment was $500 \mu \mathrm{mol} / \mathrm{l}$. The mean probenecid dose used in the study of $1.1 \mathrm{~g}$ is below that used in previous studies, for example the $2 \mathrm{~g}$ dose in the study by Reinders, et $a l^{9}$.
The decrease in serum urate level in this study was moderate, with a $29 \%$ reduction with probenecid monotherapy and a $39 \%$ reduction with combination therapy with allopurinol. Thirty-five percent of patients achieved a serum urate concentration of $<360 \mu \mathrm{mol} / \mathrm{l}$, an important target goal in the treat-to-target era. Interestingly, the authors included 15 patients (26\%) with estimated glomerular filtration rate $(\mathrm{eGFR})<50 \mathrm{ml} / \mathrm{min} / 1.73 \mathrm{~m}^{2}$, but there was no increased risk of adverse events in these patients at the doses of probenecid used in the study, and the decrease in serum urate was similar to the group with normal renal function.

It is worthwhile to compare this study with previous randomized controlled trial (RCT) evidence of the efficacy of probenecid. Reinders, et al demonstrated a $50 \%$ reduction in serum urate with a $2 \mathrm{~g}$ /day dose of probenecid after failure of allopurinol therapy (due to intolerance or failure to achieve a serum urate of $300 \mu \mathrm{mol} / 1$ or less). In addition, $65 \%$ of probenecid-treated patients in this study achieved a serum urate concentration of $300 \mu \mathrm{mol} / \mathrm{l}$ or less. Again, these patients would be regarded as having difficult-to-treat gout, with a mean serum urate concentration of $540 \mu \mathrm{mol} / 1$, and $54 \%$ having tophaceous gout ${ }^{9}$. The greater decreases in serum urate achieved by Reinders and colleagues are most likely explained by the higher probenecid doses used and the stringent environment of the RCT. Studies reporting real-world experience with medications are complementary to RCT and contribute to progress in clinical decision making.

The attitude that the RCT is king and all others must bow before it is pervasive in the medical world. In reality, each methodology has its strengths and weaknesses and truly informed "evidence-based medicine" must draw on all flavors of research study. Proponents of the evidence-based medicine approach to clinical practice often refer to a hierarchical ranking of study methodology based on internal validity; in other words, how correct study results are $^{10}$. RCT, considered the gold standard of research methodology, have been shown to have the potential to

See Probenecid as urate-lowering therapy in gout, page 872

Personal non-commercial use only. The Journal of Rheumatology Copyright (C) 2013. All rights reserved. 
exhibit considerable heterogeneity of results ${ }^{11}$. The strict inclusion and exclusion criteria of most RCT have the potential to limit the generalizability of their results to patients commonly seen in clinical practice. In addition, the rigorous and frequent study visits often create an atmosphere and time investment that is not reproducible in a busy clinical practice.

Categorizing studies according to research design and judging their validity solely on this basis is a flawed ideology: many factors other than study methodology have the potential to introduce bias, limit generalizability, and make a study less useful to practicing clinicians. Other scientific disciplines have been more willing to accept the flaws of a rigid hierarchical view of research design and we can and should learn from experience in these fields ${ }^{11}$.

A number of real-world studies of gout treatment have been reported. Allopurinol is a treatment in which the interpretation of RCT data have proven particularly difficult. In the setting of RCT, allopurinol is typically prescribed at a fixed dose of 100 to $300 \mathrm{mg}$, and is only infrequently dose-titrated based on serum urate levels, as would be the case in real-world clinical practice. This can lead to misinterpretations of the efficacy of allopurinol, if, for example, RCT of allopurinol versus febuxostat are taken at face value. RCT data on the efficacy of allopurinol typically demonstrate 21 to $46 \%$ of patients achieving a serum urate $<360$ $\mu \mathrm{mol} / 1^{12,13}$. In real-world clinical practice settings with titration of allopurinol dosage, the percentage of patients achieving target serum urate levels $<360 \mu \mathrm{mol} / 1$ increases to $58 \%$ to $100 \%^{7,14}$. Benzbromarone has been shown to have an efficacy of 78 to $92 \%$ for achieving a serum urate < $300 \mu \mathrm{mol} / 1$ in RCT, which increases to $100 \%$ in non-randomized settings ${ }^{14}$. Febuxostat has an efficacy of 53 to $94 \%$ for achieving a serum urate $<360 \mu \mathrm{mol} / \mathrm{l}$ in $\mathrm{RCT}^{12,16}$. To my knowledge there are scarce published data on the real-world efficacy of febuxostat. There are multiple subtleties in interpretation of real-world data: Findings are not as "clean" or as easy to interpret as RCT data. A cursory reading of the real-world data above would suggest that allopurinol may not be as effective as benzbromarone; however, closer examination will show that there are different degrees of "realness" and that in similar settings both agents have similar efficacy.

The integration of data from real-world clinical practice settings with RCT data provides the practicing clinician with an expanded resource of evidence with which to make optimum treatment decisions for individual patients. The increased time investment needed to properly evaluate observational studies may seem to be an obstacle; however, the time spent can be rewarding. Let's forget the old maxim: "If you find that [a] study was not randomized, we'd suggest that you stop reading it and go on to the next article" and evaluate studies on their own merits ${ }^{17}$.
RICHARD CONWAY, MB, BCh, BAO, MRCPI, Department of Rheumatology,

St. James Hospital,

Dublin, Ireland

Dr. Conway has received research funding from Roche Ireland Limited and UCB (Pharma) Ireland Limited; and honoraria from Merck and GlaxoSmithKline.

Address correspondence to Dr. Conway, Department of Rheumatology, St. James Hospital, James Street, Dublin 8, Ireland;

E-mail: drrichardconway@gmail.com

\section{REFERENCES}

1. Zhu Y, Pandya BJ, Choi HK. Prevalence of gout and hyperuricemia in the US general population: the National Health and Nutrition Examination Survey 2007-2008. Arthritis Rheum 2011;63:3136-41.

2. Becker MA, Schumacher HR, Benjamin KL, Gorevic P, Greenwald M, Fessel J, et al; Gout Natural History Study Group, Sundy JS. Quality of life and disability in patients with treatment-failure gout. J Rheumatol 2009;36:1041-8.

3. Choi HK. A prescription for lifestyle change in patients with hyperuricemia and gout. Curr Opin Rheumatol 2010;22:165-72.

4. Khanna D, Fitzgerald JD, Khanna PP, Bae S, Singh MK, Neogi T, et al. 2012 American College of Rheumatology guidelines for management of gout. Part 1: systematic nonpharmacologic and pharmacologic therapeutic approaches to hyperuricemia. Arthritis Care Res 2012;64:1431-46.

5. Khanna D, Khanna PP, Fitzgerald JD, Singh MK, Bae S, Neogi T, et al. 2012 American College of Rheumatology guidelines for management of gout. Part 2: Therapy and antiinflammatory prophylaxis of acute gouty arthritis. Arthritis Care Res 2012;64:1447-61.

6. Harrold LR, Andrade SE, Briesacher BA, Raebel MA, Fouayzi H, Yood RA, et al. Adherence with urate-lowering therapies for the treatment of gout. Arthritis Res Ther 2009;11:R46.

7. Conway R, Coughlan RJ, Carey JJ. Adherence to uric acid treatment guidelines in a rheumatology clinic. Clin Rheumatol 2012;31:1707-11.

8. Pui K, Gow PJ, Dalbeth N. Efficacy and tolerability of probenecid as urate-lowering therapy in gout; clinical experience in high prevalence population. J Rheumatol 2013;40:872-6.

9. Reinders MK, van Roon EN, Jansen TL, Delsing J, Griep EN, Hoekstra M, et al. Efficacy and tolerability of urate-lowering drugs in gout: a randomised controlled trial of benzbromarone versus probenecid after failure of allopurinol. Ann Rheum Dis 2009; 68:51-6.

10. Preventive Services Task Force. Guide to clinical preventive services: report of the U.S. Preventive Services Task Force. 2nd ed. Baltimore: Williams \& Wilkins; 1996.

11. Concato J, Shah N, Horwitz RI. Randomized, controlled trials, observational studies, and the hierarchy of research designs. N Engl J Med 2000;342:1887-92.

12. Becker MA, Schumacher HR Jr, Wortmann RL, MacDonald PA, Eustace D, Palo WA, et al. Febuxostat compared with allopurinol in patients with hyperuricemia and gout. N Engl J Med 2005;353:2450-61.

13. Becker MA, Schumacher HR, MacDonald PA, Lloyd E, Lademacher C. Clinical efficacy and safety of successful longterm urate lowering with febuxostat or allopurinol in subjects with gout. J Rheumatol 2009;36:1273-82.

14. Perez-Ruiz F, Alonso-Ruiz A, Calabozo M, Herrero-Beites A, García-Erauskin G, Ruiz-Lucea E. Efficacy of allopurinol and benzbromarone for the control of hyperuricaemia. A pathogenic approach to the treatment of primary chronic gout. Ann Rheum Dis 1998;57:545-9. 
15. Reinders MK, Haagsma C, Jansen TL, van Roon EN, Delsing J, van de Laar MA, et al. A randomised controlled trial on the efficacy and tolerability with dose escalation of allopurinol 300-600 mg/day versus benzbromarone $100-200 \mathrm{mg} / \mathrm{day}$ in patients with gout. Ann Rheum Dis 2009;68:892-7.

16. Becker MA, Schumacher HR Jr, Wortmann RL, MacDonald PA, Palo WA, Eustace D, et al. Febuxostat, a novel nonpurine selective inhibitor of xanthine oxidase: a twenty-eight-day, multicenter, phase II, randomized, double-blind, placebo-controlled, dose-response clinical trial examining safety and efficacy in patients with gout. Arthritis Rheum 2005;52:916-23.

17. Sackett DL, Richardson WS, Rosenberg W, Haynes RB.

Evidence-based medicine: how to practice and teach EBM. New York: Churchill Livingtone; 1997.

J Rheumatol 2013;40:755-7; doi:10.3899/jrheum.130113 